
\title{
25 Research Square \\ Using Bronchoalveolar Lavage Fluid for Active Pulmonary Tuberculosis Laboratory Diagnosis
}

\section{Mei Han}

Chongqing Public Health Medical Center

Jian-Qiong Guo

Southwest Hospital

\section{Xiao-Feng Yan}

Chongqing Public Health Medical Center

\section{Wu-Yang Yue}

Chongqing Public Health Medical Center

\section{Qian Qiu}

Chongqing Public Health Medical Center

\section{Le-Le Wang}

Chongqing Public Health Medical Center

\section{Qiang-Zhong Sun}

Chongqing Public Health Medical Center

\section{Ling Jiang}

Chongqing Public Health Medical Center

\section{Tong-Xin Li}

Chongqing Public Health Medical Center

\section{You-Ming Fan}

Ba'nan District People's Hospital, Chongqing,China

\section{Song Yang ( $\square$ Yangsong5@aliyun.com )}

Chongqing Public Health Medical Center

\section{Research Article}

Keywords: bronchoalveolar lavage fluid (BALF), MycobacteriumTuberculosis (MTB), active pulmonary tuberculosis (APTB), diagnosis yields

Posted Date: June 1st, 2021

DOl: https://doi.org/10.21203/rs.3.rs-557017/v1

License: (9) This work is licensed under a Creative Commons Attribution 4.0 International License. Read Full License 
Page $2 / 12$ 


\section{Abstract}

Objective: To evaluate the diagnostic efficiency of bronchoalveolar lavage fluid (BALF) for MycobacteriumTuberculosis (MTB) infection using laboratory methods.

Methods: A retrospective study was conducted in patients diagnosed with active pulmonary tuberculosis (APTB) and lacking sputum quality/quantity. BALF collected during the operation processes of Electric bronchoscopy were tested using Ziehl-Neelsen staining acid-fast bacilli smear microscopy (Z-N-AFB-SM),

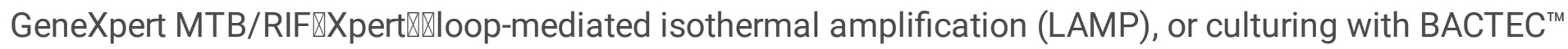
Mycobacterial Growth Indicator Tube ${ }^{\mathrm{TM}} 960$ (MGIT). Chi-square test was used for statistic analysis.

Results: 331 suspected APTB patients were enrolled in this study. 224 of them were sputum-scarce. 89 were sputum-sufficient andnegative in both Z-N-AFB-SM and MGIT 960 testing. Of the sputum sufficient patients, BALF-testing confirmed APTB diagnosis in 20.2\% (18/89) via Z-N-AFB-SM, and 53.0\% (35/89) via MGIT. The total positive rates of BALF testing via four aforementioned methods were $18.2 \%(57 / 313)$, $66.4 \%(168 / 253), 61.0 \%$ (83/136) and 48.2\% (140/290) respectively. The positive rate of MTB discovered in BALF collected by well-trained respiratory physicians are significantly higher than those collected by anesthetists $(X 2=22.48, P<0.01)$. Total adverse events incidence of BAL was $1.9 \%(6 / 313)$.

Conclusion: BALF has a similar sensitivity and specificity for APTB laboratory diagnosis. It can be used as a complementary diagnostic method for APTB when sputum availability is poor. The proficiency of BALF collection is an important factor affecting the detection results.

\section{Introduction}

Mycobacterium tuberculosis (MTB) culture is recognized as the gold-standard of laboratory method for tuberculosis (TB) infection diagnosis, however, it is difficult to perform when patients sputum availability is poor.

TB is an ancient infectious disease caused by bacillus MTB, which usually infect the lung and cause pulmonary tuberculosis (PTB). Globally, molecular diagnosis methods are strongly proposed by WHO in recent years. Despite the progresses in diagnostics methods, a considerable proportion of TB cases reported were clinically diagnosed rather than laboratory confirmed. In 2016 , for example, only $57 \%$ of the reported PTB cases were bacteriologically confirmed ${ }^{[1]}$. In China, unfortunately, it is estimated to be $30 \%$ [2], much lower than world average. Therefore, Chinese government has set up the strategy goal of improving PTB pathologic diagnostic rate to over $50 \%$ by 2020 in the "13th Five-Year National Plan for Tuberculosis Prevention and Control" [3].Corresponding to this, the Chinese National PTB diagnosis standards were revised on May 1st, 2017. Currently, one of the challenges for the laboratory diagnosis of PTB lies in obtaining quality clinical samples, especially for sputum-scarce patients. However, bronchoalveolar lavage(BAL)is a safe and well-tolerated sampling technique for bronchoalveolar cell ${ }^{[4]}$, 
and bronchoalveolar lavage fluid (BALF) obtained during the bronchoscopy is available for PTB laboratory diagnosis ${ }^{[5]}$. However, published data about PTB diagnosis yield via BALF is limited.

\section{Methods And Material}

A retrospective evaluation within 16 months (from January 2017 to April 2018) was conducted. Bronchoscopy and BAL were performed for 313 patients suspected of PTB who either had negative sputum smears for acid-fast bacilli (AFB) or could not grow on liquid medium.

Patients were diagnosed as APTB if they met WHO criteria ${ }^{[6]}$. Those with sputum smear-negative pulmonary tuberculosis (SSN-PTB) and sputum culture-negative patients (SCN-PTB) were enrolled.

All patients accepted the following examinations: electrocardiogram, pulmonary function test, chest CT scanning, prothrombin time test, and routine blood test. If no contraindication for bronchoscopy existing, flexible electric bronchoscopy (Pentax/EB-1530T3, Japan) for trans-nasal or transoral intubation with 2\% lignocaine local injections was conducted after six hours fasting, according to the procedure requirements. $20-30 \mathrm{ml}$ of normal saline was instilled for bronchial washing through the working channel, and then was aspirated into different sterile plastic containers.

BALF samples were immediately brought to the laboratory, and analyzed with Ziehl-Neelsen staining acidfast bacilli smear microscopy (Z-N-AFB-SM), Xpert MTB/RIF( Xpert, Cepheid, Sunnyvale, CA, USA), loopmediated isothermal amplification (LAMP, Eiken Chemical Co., Ltd, Tokyo Japan), and culture via BACTEC $^{\text {TM M Mycobacterial Growth Indicator Tube }}{ }^{\text {TM }} 960$ (MGIT, Becton Dickinson Diagnostic Systems, Sparks, MD, USA). Operations and quality controls strictly followed the standard procedure ${ }^{[7][8,9]}$. M. tuberculosis H37Rv provided by the National Tuberculosis Reference Laboratory (Beijing, China CDC) acted as reference strains.

All patients were tracked for 24-48 hours for adverse events after bronchoscopy, and any symptoms such as pneumothorax, hemorrhage, infection or cardiac arrhythmias were recorded. Bronchoscope was thoroughly cleaned with sterilized water by immersing in $2 \%$ glueraldehyde for one hour ${ }^{[4,10]}$. Anesthetists and well trained respiratory physicians performed the operations.

Suspected APTB diagnosis would be confirmed if any one of the laboratory testing mentioned above was positive.

Positive rate of each test was calculated respectively. Chi-square tests for statistical analysis were performed using the SPSS Statistical 20.0 software(Armonk, NY, USA) ${ }^{[11]}$. A p-value inferior to 0.05 was considered that the difference is significant.

\section{Results}


Total 313 suspected APTB patients were enrolled. 71.5\% (224/313) were sputum-scarce. 28.5\% (89/313) were sputum-sufficient and negative in Z-N-AFB-SM and MGIT 960, and their sputum was collected for further tests. Patient age ranged from 13-80 (mean: 34$)$, and 51.1\% (160/313) were males. All sputumscarce patients accepted flexible electronic bronchoscopy and the BALF were collected (Fig. 1).

89 suspected APTB patients who were sputum-sufficient accepted smearing via Z-N staining and liquid culture via MGIT using both samples of sputum and BALF. BALF-testing confirmed 18 suspected patients (20.2\%) via Z-N-AFB-SM, and 35(53.0\%) were confirmed according to liquid culture via MGIT 960 (Table 1). The difference between sputum and BALF confirmation rate is significant $(X 2=20.02,60.69$, $P<0.01)$.

Table 1

BALF and sputum for APTB diagnosis via two methods

\begin{tabular}{|lllll|}
\hline Sample type & Sample size & Positive (rate) & Sample size & Positive (rate) \\
\hline & Z-N-AFB-SM & & liquid culture via MGIT 960 \\
\hline Sputum & 89 & 0 & 89 & 0 \\
\hline BALF & 89 & $18(20.2 \%)$ & 66 & $35(53.0 \%)$ \\
\hline
\end{tabular}

All 313 patients were divided into 2 groups, sputum-scarce and sputum-sufficient. BALF collected from both groups were tested for MTB via different methods. For the sputum-sufficient group, the diagnosis yields were 20.2\% (18/89) via Z-N-AFB-SM, 55.1\% (49/89) via Xpert, 57.4\%(31/54) via LAMP, 53.0\% (35/66) via MGIT. For the sputum-scarce group, the diagnosis yields were $17.4 \%(39 / 224), 72.5 \%$ (119/164), 63.4\% (52/82) and 46.9\% (105/224) respectively (Table 2). Chi square tests suggest that there were no significant difference between the two groups by using Z-N-AFB-SM, LAMP, and culture $(\chi 2=0.56$, $0.49,0.77, P>0.05)$, but were significant by using Xpert $(\chi 2=7.92, P<0.05)$. In our study, the total positive rate using BALF was18.2\% (57/313) by method of Z-N-AFB-SM, $66.4 \%(168 / 253)$ by method of Xpert,61.0\% (83/136) by method of LAMP and $48.2 \%$ by method of MGIT respectively. The three methods were all significant higher than Z-N-AFB-SM $(X 2=119.30,60.80,6.57, P \leq 0.01)$. Xpert had the highest diagnosis yield, the difference between Xpert and MGIT was significant $(\chi 2=25.60, P<0.05)$. LAMP had the second highest diagnosis yield, the difference between LAMP and MGIT was significant too $(\mathrm{X} 2=$ $6.57, P<0.05)$. But the diagnosis yield between $X p e r t$ and LAMP were similar $(\chi 2=2.158, P>0.05)$. 
Table 2

Efficiency of BALF for PTB Laboratory Diagnosis

\begin{tabular}{|llllll|}
\hline \multirow{2}{*}{$\begin{array}{l}\text { Sample } \\
\text { type }\end{array}$} & Symptoms & \multicolumn{4}{l}{ Positive Rate(\%,Positive/sample size) } \\
\cline { 3 - 6 } & & Z-N-AFB & Xpert & LAMP & MGIT \\
& & - SM & & & \\
\hline BALF & APTB who were sputum-sufficient & 20.2 & 55.1 & 57.4 & 53.0 \\
& & $18 / 89$ & $49 / 89$ & $31 / 54$ & $35 / 66$ \\
\cline { 2 - 6 } & APTB who were sputum-scarce & 17.4 & 72.5 & 63.4 & 46.9 \\
& & $39 / 224$ & $119 / 164$ & $52 / 82$ & $105 / 224$ \\
\cline { 2 - 6 } & & 0.339 & 7.924 & 0.494 & 0.774 \\
\hline & & 0.561 & 0.005 & 0.482 & 0.379 \\
\hline
\end{tabular}

Experienced respiratory physicians who have acquired professional training of electronic bronchoscopy as well as BAL operation and anesthetists who were not well trained operated the BALF collection. After a serial of laboratory tests mentioned above, the results suggest that the positive rate of MTB discovered in BALF collected by respiratory physicians were significantly higher than that collected by anesthetists ( $\mathrm{X} 2$ $=22.48, P<0.01$ ). However the total positive rate of MTB discovered in BALF was $66.5 \%$ (Table 3).

Table 3

Positive rate of BALF collected by different doctor

\begin{tabular}{|llllll|}
\hline BALF collector & $\begin{array}{l}\text { Well-trained for } \\
\text { BAL }\end{array}$ & $\begin{array}{l}\text { Sample } \\
\text { type }\end{array}$ & $\begin{array}{l}\text { Sample } \\
\text { size }\end{array}$ & Positive & $\begin{array}{l}\text { Positive rate } \\
\text { (\%) }\end{array}$ \\
\hline $\begin{array}{l}\text { Respiratory } \\
\text { physician }\end{array}$ & Yes & BALF & 120 & 99 & 82.5 \\
\hline Anesthetist & No & BALF & 193 & 109 & 56.5 \\
\hline Total & & BALF & 313 & 208 & 66.5 \\
\hline
\end{tabular}

Tuberculosis focal spreading outside its original site and metastasizing occurred in 4 APTB patients after bronchoscopy and BAL. Another 2 patients got fever within 1-2 days after bronchoscopy, and the pulmonary foci increased suddenly. Further sputum culture results identified that Pseudomonas aeruginosa caused this secondary-acquired pneumonia. They all cured after receiving standard antituberculosis or anti- infection treatments. Total adverse events incidence was $1.9 \%(6 / 313)$.

\section{Discussion}


BALF has a similar sensitivity and specificity for APTB laboratory diagnosis. So far, culturing of MTB is still deemed as the gold standard for the diagnosis of TB ${ }^{[12]}$. In 2013, WHO made a revision to TB diagnostic criteria that the positive results tested based on molecular methods are equivalent to the positive results tested by traditional bacteriological methods. BAL was initially used to rinse the bronchial tree with saline in 1970. It evolved to a diagnostic tool in India in $1994^{[13]}$. By sampling bronchopulmonary cells and epithelial lining liquid, it can accurately diagnose all kinds of infectious and tumor diseases. It is superior to the invasive techniques such as needle aspiration biopsy and thoracoscopy, and can be used to diagnose sputum-negative TB at early stage ${ }^{[4]}$. The positive rate of TB diagnosis by BALF is $87 \%{ }^{[14]}$, and for sputum smear negative PTB it reaches $68.2 \%{ }^{[15]}$. A total of 1214 tuberculosis patients detected by BALF from 9 studies showed that the sensitivity and specificity of BALF test are $54 \%$ (95\% Cl: $48 \%-59 \%)$ and $97 \%(95 \% \mathrm{Cl}: 95 \%-98 \%)$ respectively ${ }^{[16]}$.

Complementary methods for sputum-scarce APTB are GA collection for three consecutive day, which usually requires hospital admissions and BALF collections, and may not be achievable in every setting ${ }^{[17]}$. BAL is a valuable method of respiratory tract investigation ${ }^{[18]}$..In our study, the results disclosed that the BALF has a significantly higher yield than that of sputum. For those suspected APTB with negative results using sputum both tested by Z-N-AFB-SM and by culture, $20.2 \%(18 / 89)$ and $53.0 \%(35 / 66)$ were confirmed when tested using BALF. However, there was no significant difference between sputumsufficient and scarce APTB by using BALF, the diagnosis yields of the two groups appeared consistence.

BALF has different diagnosis yield using different testing methods. In our study, we found AFB stain was positive in $18.2 \%(\mathrm{~N}=313)$ of BALF via Z-N-AFB-SM, and positive liquid culture was $48.2 \%(\mathrm{~N}=290)$ in BALF via MGIT 960. Our positive rate of Z-N-AFB-SM was slightly lower than other study reported, but the positive rate of culture based on liquid medium was significantly higher than other report based on Lowenstein-Jensen ${ }^{[19]}$. The reason may be due to the skill proficiency of the operators as well as the different culture medium.

The progress in nucleic acid amplification technology has led to a breakthrough in early detection of pulmonary tuberculosis, which is remarkably superior to traditional sputum smear test. FDA has approved commercial nucleic acid diagnosis kits for tuberculosis laboratory diagnosis, and both BALF and sputum are recommended. BALF can increase the MTB nucleic acid positive rate of SSN-PTB ${ }^{[9]} .25$ studies were reviewed, and the sensitivity/specificity of tuberculosis laboratory diagnosis method by Xpert MTB/RIF and LAMP was $89 \% / 98 \%$ and $93 \% / 94 \%$ respectively ${ }^{[20]}$. The sensitivity of BALF for Xpert and smear microscopy was $80 \%-92.3 \%$ and the specificity was $95.8 \%-98.9 \%$ [21-23] [22] [23], respectively. Among the SSN-PTB, the sensitivity and specificity of BALF tested by Xpert were $60 \%$ and $98 \%$. BALFXpert could be used as a substitute for transbronchial lung biopsy in sputum-scarce and SSN-PTB [24]. BALF being used via Xpert MTB/RIF for rapid laboratory diagnosis in high TB burden countries can promote TB treatments ${ }^{[22]}$ [23]. LAMP is another nucleic acid amplification technology that has been used for rapid diagnosis of TB clinically. WHO recommends LAMP as an alternative method to microscope for the diagnosis of PTB in adults. In our study, the positive yield using BALF was $66.4 \%$ 
(168/253) and 61.0\% (83/136) by method of Xpert MTB/RIF and LAMP respectively. Both were significant higher than Z-N-AFB-SM and culture. On the other hand, when comparing the BALF positive yields between the 2 groups patients of sputum-scarce and sufficient, there was no significance, which means BALF for APTB diagnosis are stable and repeatable.

The quality of BALF may be an important contributing factor for its diagnosis yield. BALF collected by well-trained operators had significant higher positivity. Probably attributed to this reason, there were unavoidable adverse events happened. However, it was reported that no significant adverse event was noted for patients or health-care staff recently ${ }^{[25]}$.

\section{Conclusion}

The quality of BALF may be an important contributing factor for its diagnosis yield. BALF collected by well-trained operators had significant higher positivity. Probably attributed to this reason, there were unavoidable adverse events happened. However, it was reported that no significant adverse event was noted for patients or health-care staff recently.

\section{List Of Abbreviations}

\begin{tabular}{|c|c|}
\hline Mycobacterium tuberculosis & MTB \\
\hline tuberculosis & TB \\
\hline pulmonary tuberculosis & PTB \\
\hline bronchoalveolar lavage & BAL \\
\hline bronchoalveolar lavage fluid & BALF \\
\hline acid-fast bacilli & AFB \\
\hline sputum smear-negative pulmonary tuberculosis & SSN-PTB \\
\hline sputum culture-negative patients & SCN-PTB \\
\hline Ziehl-Neelsen staining acid-fast bacilli smear microscopy & Z-N-AFB-SM \\
\hline Xpert MTB/RIF & Xpert \\
\hline BACTEC ${ }^{\text {TM }}$ Mycobacterial Growth Indicator Tube ${ }^{\text {TM }} 960$ & MGIT \\
\hline
\end{tabular}

\section{Declarations}

\section{Ethics approval and consent to participate}

The study was approved by the Ethics Board of Chongqing Public Health Medical Center. 
Not applicable.

\section{Availability of data and materials}

All data generated or analyzed during this study are included in this published article.

\section{Competing interests}

The authors declare that they have no competing interests.

\section{Funding}

This work was supported by Chongqing Technology Innovation and Application Development Special Project (No.cstc2019jscx-msxmX0152); Medical Research Project of Chongqing Science and Technology Commission and Health Commission in 2019 (No.2019MSXM065).

\section{Authors' contributions}

SY, QZ-S, LJ, JQ-G, YM-F and XF-Y designed the study. MH, SY, TX-L and WY-Y analyzed the data.MH, JQG, QQ and SY drafted the manuscript. SY, QZ-S, LL-W and LJ provided clinical data. TX-L and WY-Y operated the lab testing. SY supervised the study. All authors read and approved the final manuscript.

\section{Acknowledgements}

We would like to thank all study participants who were involved and contributed to the data collection. We thank editors and reviewers for their warm works and valuable comments.

We especially thank Prof. Shenjie Tang, Beijing Chest Hospital Affliated to Capital Medical University, Beijing, China. for his critical evaluation on the article,

\section{References}

1. World Health Organization. (2017). Global tuberculosis report 2017. World Health Organization. https://apps.who.int/iris/handle/10665/259366.

2. Hagiwara $\mathrm{E}$, Katano T, Isomoto $\mathrm{K}$, et al. Clinical characteristics and early outcomes of patients newly diagnosed with pulmonary Mycobacterium avium complex disease[J]. Respir Investig. 2019, 57(1): 54-59.

3. China. G O O T. The office of the State Council on the issuance of the notice of National tuberculosis prevention and control program in 13th Five-Year. State Office issued [2017][J]. 2017.

4. Radha S, Afroz T, Prasad S, et al. Diagnostic utility of bronchoalveolar lavage[J]. J Cytol. 2014, 31(3): 136-138.

5. Mondoni M, Repossi A, Carlucci P, et al. Bronchoscopic techniques in the management of patients with tuberculosis[J]. Int J Infect Dis. 2017, 64: 27-37. 
6. Yan L, Tang S, Yang Y, et al. A Large Cohort Study on the Clinical Value of Simultaneous Amplification and Testing for the Diagnosis of Pulmonary Tuberculosis[J]. Medicine (Baltimore). 2016, 95(4): e2597.

7. Yan L, Zhang Q, Xiao H. Clinical diagnostic value of simultaneous amplification and testing for the diagnosis of sputum-scarce pulmonary tuberculosis[J]. BMC Infect Dis. 2017, 17(1): 545.

8. Gelaw B, Shiferaw Y, Alemayehu M, et al. Comparison of loop-mediated isothermal amplification assay and smear microscopy with culture for the diagnostic accuracy of tuberculosis[J]. BMC Infect Dis. 2017, 17(1): 79.

9. Ramírez P, Valencia M, Torres A. Bronchoalveolar lavage to diagnose respiratory infections[J]. Semin Respir Crit Care Med. 2007, 28(5): 525-533.

10. Gopathi N R, Mandava V, Namballa U R, et al. A Comparative Study of Induced Sputum and Bronchial Washings in Diagnosing Sputum Smear Negative Pulmonary Tuberculosis[J]. J Clin Diagn Res. 2016, 10(3): C7-C10.

11. Le Palud P, Cattoir V, Malbruny B, et al. Retrospective observational study of diagnostic accuracy of the Xpert ${ }^{\circledR}$ MTB/RIF assay on fiberoptic bronchoscopy sampling for early diagnosis of smearnegative or sputum-scarce patients with suspected tuberculosis[J]. BMC Pulm Med. 2014, 14: 137.

12. Pang $C$, Wu Y, Wan C, et al. Accuracy of the Bronchoalveolar Lavage Enzyme-Linked Immunospot Assay for the Diagnosis of Pulmonary Tuberculosis: A Meta-analysis[J]. Medicine (Baltimore). 2016, 95(12): e3183.

13. Hoffman A M. Bronchoalveolar lavage: sampling technique and guidelines for cytologic preparation and interpretation[J]. Vet Clin North Am Equine Pract. 2008, 24(2): 423-435.

14. Ullmann A J, Aguado J M, Arikan-Akdagli S, et al. Diagnosis and management of Aspergillus diseases: executive summary of the 2017 ESCMID-ECMM-ERS guideline[J]. Clin Microbiol Infect. 2018, 24 Suppl 1: e1-e38.

15. Kumar R, Singh M, Gupta N, et al. Bronchoscopy in immediate diagnosis of smear negative tuberculosis[J]. Pneumonol Alergol Pol. 2014, 82(5): 410-414.

16. Tian P, Shen Y, Wang Y, et al. Diagnostic value of nucleic acid amplification tests on bronchoalveolar lavage fluid for smear-negative pulmonary tuberculosis: a meta-analysis[J]. Biosci Rep. 2015, 35(4).

17. Wang Hw J F K M. Bronchoscopic Interventional Treatment[M]. The second edition ed. People's Medical Publishing House, 2017: 64-115.

18. Domagala-Kulawik J. The relevance of bronchoalveolar lavage fluid analysis for lung cancer patients[J]. Expert Rev Respir Med. 2020, 14(3): 329-337.

19. çakır $E$, özdemir $A$, Daşkaya $H$, et al. The value of nasopharyngeal aspirate, gastric aspirate and bronchoalveolar lavage fluid in the diagnosis of childhood tuberculosis[J]. Turk J Pediatr. 2018, 60(1): 10-13.

20. Yan L, Xiao H, Zhang Q. Systematic review: Comparison of Xpert MTB/RIF, LAMP and SAT methods for the diagnosis of pulmonary tuberculosis[J]. Tuberculosis (Edinb). 2016, 96: 75-86. 
21. Le Palud P, Cattoir V, Malbruny B, et al. Retrospective observational study of diagnostic accuracy of the Xpert ${ }^{\circledR}$ MTB/RIF assay on fiberoptic bronchoscopy sampling for early diagnosis of smearnegative or sputum-scarce patients with suspected tuberculosis[J]. BMC Pulm Med. 2014, 14: 137.

22. Lu Y, Zhu Y, Shen N, et al. Evaluating the diagnostic accuracy of the Xpert MTB/RIF assay on bronchoalveolar lavage fluid: A retrospective study[J]. Int J Infect Dis. 2018, 71: 14-19.

23. Barnard D A, Irusen E M, Bruwer J W, et al. The utility of Xpert MTB/RIF performed on bronchial washings obtained in patients with suspected pulmonary tuberculosis in a high prevalence setting[J]. BMC Pulm Med. 2015, 15: 103.

24. Mok Y. Do we need transbronchial lung biopsy if we have bronchial[J]. Int J Tuberc Lung Dis. 2016, 20(5): 619-624.

25. Taton O, Papleux E, Bondue B, et al. Role of the Bronchoalveolar Lavage in Noncritically III Patients during the SARS-CoV-2 Epidemic[J]. Pulm Med. 2020, 2020: 9012187.

\section{Figures}

\section{Active PTB patients confirmed by clinical diagnosis $(\mathbf{n}=331$ )}

\section{Sputum-scarce patients $(\mathrm{n}=\mathbf{2 2 4})$}

Figure 1

Flow chart of the study

Page 12/12 